\title{
POST CABG REHABILITATION \\ Comprehensive cardiac rehabilitation and quality of life in coronary artery bypass graft patients
}

\section{L. van Rooy and Y. Coopoo}

Faculty of Health Sciences, Department of Sport and Movement Studies, University of Johannesburg, Johannesburg, South Africa.

Address for correspondence:

Ms Lynn van Rooy

Department of Sport and Movement Studies

Faculty of Health Sciences

University of Johannesburg

Doornfontein Campus

$\mathrm{PO}$ Box 524

Auckland Park

Johannesburg

2006

South Africa

\section{Email:}

lynnvr@uj.ac.za

\section{INTRODUCTION}

Coronary artery bypass graft (CABG) has been identified as an important surgical tool in the treatment of coronary artery disease (CAD) and has been used for nearly 50 years. ${ }^{(1,2)}$ CABG surgery substantially improves symptoms in more than $90 \%$ of patients who undergo this treatment, and is favoured over other surgical interventions such as percutaneous transluminal coronary angioplasty (PTCA). ${ }^{(3)}$ This author further states that despite this evidence patients prefer not to undergo CABG surgery because of the fear of complications, as well as of physical and mental debilitation owing to invasive surgery. This is where rehabilitative personnel have a significant role to play as the overall success of CABG surgery, although a complex operation, is as much dependent on the postoperative rehabilitation and recovery period as the intra-operative clinical period. (4) The notion that rehabilitation is offered as a single service is outdated. A comprehensive CR programme should typically be multidisciplinary in approach, and should be needbased and cost-effective combining exercise training, lifestyle modification and behavioural therapy.(5,6) More importantly, patient education should accompany CR programmes, so that patients can learn to manage their health, as very little attention is given to the enhancement of knowledge and assessment of QoL during patient care. The purpose of measuring healthrelated QoL (HRQoL) is to gain an insight into how individuals view the changes that have happened in their health and how

\section{ABSTRACT}

The overall success of coronary artery bypass graft (CABG) surgery is much dependent on the postoperative rehabilitation. Cardiac rehabilitation (CR) programmes should be comprehensive and multifactorial to enhance the education and quality of life of the patient.

This was a quasi-experimental and longitudinal intervention study to which 18 patients who had undergone CABG surgery were referred. Two questionnaires were administered and patients participated in a 12 -week exercise programme.

Significant improvements were noted in waist circumference $(99.7 \pm 8.3$ to $97.8 \pm 8.8 ; p=0.042)$, body fat percentage $(22.7 \pm 5.3 \%$ to $20.5 \pm 4.9 \% ; p=0.000)$, resting systolic blood pressure $(125.7 \pm 13.7 \mathrm{mmHg}$ to $119.7 \pm$ $8.9 \mathrm{mmHg} ; \mathrm{p}=0.000)$, stairs climbed $(3.6 \pm 3.5$ flights to $5.9 \pm 4.4$ flights; $p=0.003$ ), duration of exercise per week ( $329 \pm 383.7 \mathrm{~min} . w k-I$ to $527 \pm 482.7 \mathrm{~min} . w k-I ; p=0.00 I$ ), kilocalories expended per session $(827 \pm 765 \mathrm{kcal}$ to $1173 \pm 699 \mathrm{kcal} ; \mathrm{p}=0.00 \mathrm{I}$ ), kilocalories expended per week ( $2550 \pm 858 \mathrm{kcal}$ to $3996 \pm$ I $849 \mathrm{kcal} ; p=0.000$ ), chair sit-and-reach $(0.7 \pm 3.4 \mathrm{~cm}$ to $I .3 \pm 3.4 \mathrm{~cm} ; \mathrm{p}=0.00 \mathrm{I})$, up and go (6.0 $\pm 1.3 \mathrm{sec}$ to $5.1 \pm 1.2 \mathrm{sec} ; \mathrm{p}=0.002)$, chair stand ( $13.4 \pm 2.3$ to $16.2 \pm 2.3 ; p=0.000)$, maximum workload $(82 \pm 23.0$ watt to $110 \pm 29.4$ watt; $p=0.000)$, maximum duration (08:35 \pm 02:4 I min:sec to $\mathrm{II}: 57 \pm$ 03:5 I min:sec; $\mathrm{p}=0.000)$, physical component score (38.8 \pm 6.1 to $52.1 \pm 4.2 ; p=0.000)$ and mental component score $(49.5 \pm 8.5$ to $56.9 \pm 3.1 ; p=0.002)$.

The findings of this study support the role of $C R$ encompassing lifestyle intervention, education and psychological support in promoting a faster recovery of health and desired performance of the CABG patient. SAHeart 2017;14:154-160

these changes have affected their lives. The assessment of QoL has thus proven beneficial for CABG patients to address emotional distress, and limitations due to physical pain and decreased social functioning. ${ }^{(7)}$ However, despite the importance of QoL, patient-reported outcomes are not measured in most cardiovascular clinical trials.

Regrettably, rehabilitation programmes have been apt to start several months after acute MI, missing what may be a crucial period for rehabilitation. ${ }^{(8)}$ This highlights the importance of 
assessing the clinical efficacy of rehabilitation in chronic diseases, not only in terms of mortality and morbidity, but also in terms of HRQOL. ${ }^{(9)}$ Therefore, the aim of this study was to highlight the importance and efficacy of comprehensive CR programmes which include exercise as well as nutritional and psychological interventions. This article aims to introduce individuals to the importance of physical activity in improving QoL, as well as encourages the incorporation of multi-dimensional CR across a single discipline.

\section{MATERIAL AND METHODS}

\section{Research design}

This research study made use of 2 designs, namely quasiexperimental and longitudinal intervention, and is quantitative in nature. It explored the effects of exercise programmes, coupled with lifestyle modification, including nutrition knowledge, which is not reported on in this article, and psychosocial health, of CABG patients.

\section{Study population}

Testing and implementation of this research took place at 2 private biokinetic practices in Johannesburg. Each of the practice owners signed a consent form, permitting testing and rehabilitation to take place at these facilities. Eighteen patients were recruited after undergoing CABG surgery. Although this is a small sample size, a previous study conducted on participants of a similar mean age identified lack of motivation, lack of time and depressive symptoms as barriers to participation in CR. ${ }^{(10)}$ Upon meeting the inclusion criteria and obtaining clearance from the cardiothoracic surgeon and/or cardiologist, patients were referred to the study. The inclusion criteria required patients to present with no comorbid chronic diseases, excluding hypertension, diabetes, hypercholesterolemia or a combination of 2 or 3 of the aforementioned, as they are classified as lower risk patients compared to their counterparts with other comorbidities. Each participant served as their own control, measuring the difference in quality of life before the implementation of the exercise programme and lifestyle modification. Ethical approval to conduct health-related tests on participants was obtained from the Academic Ethics Committee of the Faculty of Health Sciences at the University of Johannesburg (AEC78/02-20II).

\section{Measuring tools and instruments}

Each participant was assessed at the pre- and post-intervention stage using identical protocols. Questionnaires were issued to participants pre- and post-intervention. The intervention phase of this study spanned 12 weeks and consisted of individualised exercise programmes, completed 3 times a week. To supplement rehabilitation, each participant received a lifestyle manual which outlined various nutritional guidelines and stress reduction tools to assist in behavioural changes.

\section{Questionnaires}

Two questionnaires formed part of this research study. The SF-36v $2^{\circledR}$ Health Survey ${ }^{(1)}$ was utilised to measure QoL and has been used in many African studies as a measuring tool.(12-14) It is suitable for administration to adults 18 years and older and can be used across age, disease and treatment groups. This questionnaire comprises 36 five-point, Likert-scale items used to measure 8 domains of HRQoL, namely physical functioning (PF), role limitations due to physical health (RP), bodily pain (BP), general health perceptions $(\mathrm{GH})$, vitality $(V T)$, social functioning (SF), role limitations due to emotional problems (RE) and mental health $(\mathrm{MH})$. (I) The information obtained on these 8 domains were further aggregated into 2 summary component measures of physical and mental health (PCS, MCS), and the norm-based score was calibrated at 50. Scoring took place by computing the information obtained from this survey into a software programme (Health Outcomes Scoring Software, version 4.5). Rehabilitation performed during the 12 week intervention was supervised by the researcher.

The Physical Activity (PA) Questionnaire, originally developed by Paffenbarger (1978), was utilised for the purpose of determining the amount of physical activity performed by participants prior to having CABG surgery and after the intervention. ${ }^{(15)}$ This questionnaire has been used widely and has proven to be a constructive tool in the clinical assessment of CAD patients who have undergone angioplasty. ${ }^{(16)}$ This questionnaire consists of 8 quantitative questions, all related to physical and recreational activity. The parameters measured by this questionnaire include the average distance walked per day, flight of stairs climbed daily and amount of physical activity performed. The scoring card lists various sport and activities, their intensities and metabolic equivalent (MET) levels. From this information, it was possible to estimate the number of kilocalories expended on a sessional and weekly basis, as well as ascertaining if behaviour regarding physical activity had changed and to what extent.

\section{Morphological, biochemical and health-related parameters}

Height, weight, body mass index (BMI), waist circumference and body fat percentage made up the morphological variables assessed in this study, and was tested at the pre- and postintervention stages. Using values from the height and weight measurements, it was possible to calculate BMI. Body fat percentage was also determined by conducting a 7-site skinfold assessment on each participant.

Heart rate (HR), systolic blood pressure (SBP), diastolic blood pressure (DBP), blood glucose and total blood cholesterol (non-fasting) were measured at the pre- and post-intervention. The HR, SBP and DBP assessed included resting, exercise and recovery measures. Blood glucose and cholesterol were screened by means of collecting capillary blood samples. 
The aerobic test utilised in this study to determine the cardiorespiratory fitness of the participants was the adapted YMCA protocol on a stationary bicycle.(17) The researcher opted to adapt this protocol by increasing the workload in increments of 3 minutes. This made it easier for pre- and postintervention comparisons, as well as intergroup comparisons, because the protocol was standardised for all participants. The chair sit-and-reach test was assessed to determine the lowerbody flexibility of the participants. The protocol followed for this test was derived from Rikli and Jones (200I), and was suitable for this group of participants as they can be classified as senior citizens. ${ }^{(18)}$ The eight foot-up-and-go test and the chair stand test was conducted to determine the neuromotor strength of participants. This is of great importance as neuromotor strength is strongly associated with the ability to perform activities of daily living (ADLs).

\section{Intervention programme}

This study comprised an intervention programme consisting of exercise prescription, nutritional guidelines and psychological advice over a 12-week period. Participants were prescribed individualised exercise programmes based on results concluded in the assessment, with a frequency of 3 times per week (low-to-moderate intensity depending on the findings in the assessment).

All exercise was supervised by an exercise therapist and any concerns regarding nutritional or psychological challenges were communicated after each exercise session, however, this was not recorded and used as part of the data analysis. Since exercise prescription should include 3 stages of progression: a starting stage, a stage of slow-to-moderate progression, and a maintenance stage, exercise programmes were updated and progressed every 4 weeks to ensure that progression took place. ${ }^{(19)}$

\section{Data analysis}

Upon completion of this study, the data collected was analysed by the University of Johannesburg's Statistical Consultation Services (STATKON). The statistical analysis was completed on SPSS (Statistical Package for Social Sciences) for Windows version 15.0 (SPSS Inc., Chicago, IL, USA). Descriptive and inferential statistics were computed and standard deviations were used in order to determine variance of the sample.

To test for normality, the Kolmogorov-Smirnov and ShapiroWilk tests were used. Two non-parametric tests were used to determine significant differences between data sets; the Friedman test is an extension of the Wilcoxon Signed Rank test and allows for the analysis of repeated-measures data if participants are assessed on 2 or more occasions.(20) The Wilcoxon Signed Rank test was done to determine the significance over 2 time periods. The level of significance was set at $5 \%(p \leq 0.05)$ for the Wilcoxon Signed Rank test. The level of significance was set at $1 \%(p \leq 0.0167)$ in instances where the Friedman test was used because the Bonferroni adjustment was applied.

\section{RESULTS}

The results section is organised in 4 subcategories, each addressing factors that have contributed to an overall improvement in QoL. The results depicted are descriptive and inferential in nature. The demographical and mean morphological data, physiological and biochemical parameters, overall physical activity, various domains of QoL, neuromotor and healthrelated fitness test results are depicted in the tables below.

\section{Demographic data (Table I)}

The average age of patients was 65.2 years. The total sample undergoing intervention after CABG consisted of 17 male participants and I female. Five were diabetic, 12 hypertensive and 8 hypercholesterolemic. Only I participant reported a smoking history and 12 had been placed on lipid-lowering therapy.

TABLE I: Demographic data and comorbid diseases of the sample $(n=18)$.

\begin{tabular}{|l|c|}
\hline & $n$ \\
\hline Age (yrs) & $65.2 \pm 8.0$ years \\
\hline Male gender & 17 \\
\hline Diabetes & 5 \\
\hline Hypertension & 12 \\
\hline Smoking history & 1 \\
\hline Hypercholesterolemia* & 8 \\
\hline Lipid lowering therapy (statin use) & 12 \\
\hline
\end{tabular}

Indicating elevated lipoprotein levels in the blood, as diagnosed by a physician.

\section{Morphological data (Table II)}

A significant reduction of $1.9 \mathrm{~cm}(-2 \%)$ in average waist circumference was observed from pre- to post-intervention ( $p=0.042$ ). Average body fat percentage significantly reduced from $22.7 \%$ at the pre-intervention stage to $20.5 \%$ at the post-intervention stage, reflecting a $10 \%$ reduction $(p=0.000)$.

TABLE II: Morphological data.

\begin{tabular}{|l|c|c|c|}
\hline & \multicolumn{2}{c}{ Mean \pm SD } & P value \\
\hline Height $(\mathrm{cm})$ & $172 \pm 8.3$ & $172 \pm 8.3$ & - \\
\hline Weight $(\mathrm{kg})$ & $87.3 \pm 11.6$ & $87.6 \pm 11.9$ & 0.573 \\
\hline BMl $\left(\mathrm{kg} / \mathrm{m}^{2}\right)$ & $29.1 \pm 3.7$ & $29.3 \pm 3.6$ & 0.397 \\
\hline Waist circumference $(\mathrm{cm})$ & $99.7 \pm 8.3$ & $97.8 \pm 8.8$ & 0.042 \\
\hline Body fat percentage $(\%)$ & $22.7 \pm 5.3$ & $20.5 \pm 4.9$ & 0.000 \\
\hline
\end{tabular}


Physiological and biochemical parameters (Table III) The Friedman test was used to assess for significance in resting systolic blood pressure (SBP). The resting SBP reduced significantly from 125.7 - $119.7 \mathrm{mmHg}$ (pre- to post-intervention).

TABLE III: Physiological and biochemical parameters.

\begin{tabular}{l|c|c|c|}
\hline & \multicolumn{2}{c}{ Mean \pm SD } & P value \\
\hline Resting HR $(\mathrm{bpm})$ & $70.6 \pm 10.5$ & $74.8 \pm 10.1$ & 0.276 \\
\hline Resting SBP $(\mathrm{mmHg})$ & $125.7 \pm 13.7$ & $119.7 \pm 8.9$ & 0.000 \\
\hline Resting DBP $(\mathrm{mmHg})$ & $76.5 \pm 6.8$ & $75.8 \pm 6.6$ & 0.802 \\
\hline Blood glucose $(\mathrm{mmol} / \mathrm{l})$ & $4.6 \pm 1.4$ & $5.9 \pm 1.7$ & 0.747 \\
\hline Blood cholesterol $(\mathrm{mmol} / \mathrm{l})$ & $4.5 \pm 1.2$ & $4.2 \pm 0.9$ & 0.122 \\
\hline
\end{tabular}

\section{Overall physical activity levels (Table IV)}

The average distance walked and flights of stairs climbed at the pre-intervention interval was increased from $1.9 \mathrm{~km}$ and 3.6 flights of stairs to $2.5 \mathrm{~km}$ and 5.9 flights of stairs, respectively. Flights of stairs climbed exhibited a significant increase $(p=0.003)$. The duration of physical activity and kilocalorie expenditure taken at the time of the pre-intervention stage was recorded at 329min.wk-I, 827kcal per session and 2 550kcal per week which increased significantly to 527 min.wk- I ( $50 \%$; $p=0.00 \mathrm{I}), \quad$ I $173 \mathrm{kcal}$ (42\%) per session, and $2550 \mathrm{kcal}$. $3996 \mathrm{kcal}(57 \%)$ per week $(p=0.00 \mathrm{I}$ and $p=0.000)$, respectively.

\section{TABLE IV: Physical activity questionnaire.}

\begin{tabular}{lcc|c}
\hline & \multicolumn{2}{c}{ Mean \pm SD } & P value \\
& Pre & Post & \\
\hline Distance walked $(\mathrm{km})$ & $1.9 \pm 1.3$ & $2.5 \pm 1.3$ & 0.082 \\
\hline $\begin{array}{l}\text { Stairs (flights) } \\
\text { Duration per week } \\
\text { (min.wk- })\end{array}$ & $3.6 \pm 3.5$ & $5.9 \pm 4.4$ & 0.003 \\
\hline Kilocalories per session (kcal) & $827 \pm 765$ & $1173 \pm 699$ & 0.001 \\
\hline Kilocalories per week (kcal) & $2550 \pm 1858$ & $3996 \pm 1849$ & 0.000 \\
\hline
\end{tabular}

\section{Quality of life (Table V)}

Physical functioning significantly increased by $74 \%$ by the time the post-intervention test was conducted. Other factors that showed significant improvements were bodily pain (49\%), general health (20.0\%), vitality (50.4\%) and social functioning (51\%). Role physical limitations, emotional problems and mental health significantly improved by $114 \%, 34 \%$, and $18 \%$ respectively. The physical and mental component summary scores reflected significant increases of $34 \%$ and I5\% respectively.

\section{TABLE V: Quality of life (SF-36v2 Health Survey).}

\begin{tabular}{|c|c|c|c|}
\hline & \multicolumn{2}{|c|}{ Mean \pm SD } & \multirow[t]{2}{*}{$P$ value } \\
\hline & Pre & Post & \\
\hline Physical functioning (\%) & $47.8 \pm 21.0$ & $83.1 \pm 12.9$ & 0.000 \\
\hline Role Physical (\%) & $44.1 \pm 25.7$ & $94.4 \pm 9.3$ & 0.000 \\
\hline Bodily pain (\%) & $52.7 \pm 24.2$ & $78.6 \pm 15.1$ & 0.001 \\
\hline General health (\%) & $64.7 \pm 20.5$ & $77.6 \pm 8.3$ & 0.041 \\
\hline Vitality (\%) & $48.3 \pm 15.9$ & $72.6 \pm 12.0$ & 0.001 \\
\hline Social functioning (\%) & $60.4 \pm 23.2$ & $100.0 \pm 14.7$ & 0.001 \\
\hline Role emotional (\%) & $74.5 \pm 23.8$ & $99.5 \pm 2.0$ & 0.001 \\
\hline Mental health (\%) & $74.7 \pm 16.1$ & $87.8 \pm 7.7$ & 0.005 \\
\hline $\begin{array}{l}\text { Physical component } \\
\text { score (\%) }\end{array}$ & $38.8 \pm 6.1$ & $52.1 \pm 4.2$ & 0.000 \\
\hline $\begin{array}{l}\text { Mental component } \\
\text { score }(\%)\end{array}$ & $49.5 \pm 8.5$ & $56.9 \pm 3.1$ & 0.002 \\
\hline
\end{tabular}

Neuromotor test results (Table VI)

The chair sit-and-reach test showed a significant increase of $75 \%$ comparing the pre- and post-intervention test data. The average time taken to complete the up-and-go test significantly reduced (-21\%) from 6.0 seconds -5.1 seconds in the post-intervention stage. Participants significantly improved their average total chair stands from 13.4 - 16.2 (14\%) postintervention

TABLE VI: Neuromotor test results.

\begin{tabular}{|c|c|c|c|}
\hline & \multicolumn{2}{|c|}{ Mean \pm SD } & \multirow[t]{2}{*}{$P$ value } \\
\hline & Pre & Post & \\
\hline Chair sit-and-reach (cm) & $0.7 \pm 3.4$ & $1.3 \pm 3.4$ & 0.001 \\
\hline Up-and-go (sec) & $6.0 \pm 1.3$ & $5.1 \pm 1.2$ & 0.002 \\
\hline Chair stand & $13.4 \pm 2.3$ & $16.2 \pm 2.3$ & 0.000 \\
\hline
\end{tabular}

Health-related fitness test results (Table VII)

The average maximum workload significantly increased from 82 - 110 watts. The average maximum duration cycled also significantly increased from 08:35 - | |:57min:sec.

TABLE VII: Health-related fitness test results.

\begin{tabular}{|c|c|c|c|}
\hline & \multicolumn{2}{|c|}{ Mean \pm SD } & \multirow[t]{2}{*}{$P$ value } \\
\hline & Pre & Post & \\
\hline Max workload (watts) & $82 \pm 23.0$ & $110 \pm 29.4$ & 0.000 \\
\hline Max duration (min:sec) & $08: 35 \pm 02: 41$ & $|1: 57 \pm 03: 5|$ & 0.000 \\
\hline HR (bpm) & $118 \pm 13.6$ & $121 \pm 17.7$ & 0.391 \\
\hline $\mathrm{SBP}(\mathrm{mmHg})$ & $159 \pm 19.8$ & $157 \pm 14.0$ & 0.284 \\
\hline $\mathrm{DBP}(\mathrm{mmHg})$ & $81 \pm 7.1$ & $80 \pm 6.7$ & 0.721 \\
\hline RPE & $8 \pm 0.2$ & $8 \pm 0.2$ & 0.264 \\
\hline
\end{tabular}




\section{DISCUSSION}

While all domains of HRQoL exhibited significant improvements, physical and role functioning displayed the largest improvement. This is supported by the findings in this study, with reference to the neuromotor test results, the fitness results and the PA questionnaire scores. ${ }^{(15)}$ Notably, all these components reflected significant improvements, resulting in an increase in the participants' abilities to perform ADLs. More specifically, preliminary research evidence suggests that physical activity helps reverse exercise intolerance and improves mood and QoL. (21,22) The present study yielded significant reductions in all neuromotor tests measured. These tests included the chair sit-and-reach, up-and-go, and chair stand tests, all made suitable for use by senior citizens. Seguin, et al. (20/2) conducted research on older female participants with a mean age of 63 years, noting significant improvements in all Senior Fitness Test measures after a 10 week strength training programme had been administered twice a week. ${ }^{(23)}$ Similarly, another study conducted on CABG patients to assess the effect of rehabilitation on strength and balance, yielded significant results in the chair stand and up-and-go tests. ${ }^{(24)}$ From the aforementioned studies, it is evident that neuromotor training is imperative for increasing independence, confidence and reassurance.

Regular exercise has proved to: increase muscular function and stimulate the body's ability to consume and utilise oxygen, improve the capacity of blood vessels to dilate in response to exercise, ventricular diastolic function and neurohormonal activation. (25) In a study by $\mathrm{Hsu}$, et al. (201 I) on early CR and HRQoL, the results implied that the relative increase of physical capacity is the major determinant of improved HRQOL. ${ }^{(26)}$ In the present study, a significant decline in SBP was noted between the pre- and post-intervention test which could possibly be attributed to the fact that exercising regularly reduces the control of the baroreflex and sympathetic nerve traffic.(27) Exercise impacts on the activation of the baroreceptors, which decreases the peripheral vasculature and peripheral vascular resistance, thus further lowering BP.(27) Furthermore, a previous study has indicated that mechanisms by which aerobic exercise lowers BP partly involves the effects of epinephrine and norepinephrine. ${ }^{(28)}$ Aerobic exercise reduces the blood level of norepinephrine which limits vasoconstriction of the arterioles and decreases the peripheral resistance to $\mathrm{BP}$, hence causing reductions in BP. ${ }^{(28)}$ Another possible reason for the reductions in SBP in this study could be that regular exercise also lowers HR due to activation of the parasympathetic division of the autonomic nervous system, which in turn allows the ventricles more time to fill with blood, ensuring adequate oxygen and nutrient delivery. ${ }^{(27)}$ This is consistent with the significant improvements found in the maximum workloads reached, maximum duration cycled during the aerobic test, the amount of flights of stairs climbed, duration of physical activity per week, and kilocalories expended per session and per week, as well as HRQoL, in this study. The maximum workload and maximum duration cycled were both found to increase significantly, apparent demonstrations that an increase in exercise is linked to improvements in aerobic endurance. ${ }^{(18)}$ In a study by Cadore, et al. (201 I) on elderly men, significant improvement in maximum workload were found in the aerobic training and concurrent training groups. ${ }^{(29)}$ This is noteworthy because the training was conducted over a 12-week period, 3 times a week, which is consistent with the present study. This increase in fitness could be highly attributable to the amount of flights of stairs climbed, duration of physical activity per week and kilocalories expended per week and per session, which are all components of the PA questionnaire and were found to increase significantly in the comparison between pre- and posttest. (15) At the post-test, the mean duration of physical activity taken was $527.2 \mathrm{~min} . w \mathrm{k}-\mathrm{I}$ which is equivalent to roughly 90 minutes of physical activity at least 6 days a week. Although this is consistent with the recommended frequency of exercise according to Aroesty (2010), this quantity of exercise is in excess of the current recommendation, as stated by Adams (2013), which is $150-210 \mathrm{~min}$.wk-I of aerobic and resistance exercise spread over 3 - 5 sessions. ${ }^{(30,31)}$ Additionally, the number of kilocalories expended per week at the post-test was also consistent with the recommendations of 1000 $2000 \mathrm{kcal}$ expended per week necessary for general health benefits. ${ }^{(21)}$ These findings have indicated that the quantity of exercise performed by the participants in the present study have been in line with the current recommended frequencies and intensities to reach and maintain optimal health.

While the key objective of CR is to develop and improve QoL, post-operative pain levels and ADLs are significant indicators for evaluating the changes in physical and psychological wellbeing of cardiac patients. ${ }^{(27,32)}$ Correspondingly, bodily pain, general health and vitality were also found to improve significantly. Additionally, the social functioning, role limitations due to emotional problems, and mental health domains also yielded statistically significant results, which have been previously linked to improved QoL and sense of well-being. ${ }^{(33)}$ As a result of CABG surgery, some of the most important adverse consequences include forced inactivity, pain in the chest and vein harvest site and reduction of QoL, which may all contribute to severe stress and depression. ${ }^{(27)}$ Research has indicated that non-pharmacological interventions, such as exercise training and psycho-education, have a positive physiological and psychological effect in early outpatient rehabilitation in patients who have undergone CABG surgery. ${ }^{(34)}$ 
The impact of CR on patients' weight, waist circumference and body fat was somewhat unexpected. In a study by Coopoo, Berger and Andrews (1995) on a cohort of sedentary Indian males, similar decreases in body fat percentage were seen after the intervention. ${ }^{(35)}$ Although BMI was shown to increase insignificantly in this study, attributable to the increase in body weight, the decrease in body fat percentage points toward a reduction in fat mass and an increase in lean mass.(36) The possible explanation for this is that participants engaged in a resistance training programme, which normally increases energy output. Moreover, aerobic training increases energy expenditure by activation of lipolysis and affects the reduction of body fat percentage.(28) A study conducted on postmenopausal women further indicates that reductions in weight improves the physical and mental HRQoL domains and leads to a positive change in self-perceived health status. ${ }^{(37)}$ Similarly, significant reductions in waist circumference were also observed among this group of participants. This is in contrast to a study conducted by Miyatake, et al. (2007) who concluded that a decrease in body weight corresponds with a decrease in waist circumference in Japanese men and women. ${ }^{(38)}$ Although the findings regarding body weight in this study were not expected, it has been stated that exercise without weight loss is associated with a substantial decrease in total and abdominal fat. (39) Research conducted by Staniūtè and Brožaitienè (20I0), which assessed the HRQoL in patients with coronary heart disease (CHD), established that improvements occurred over 2 years, however, the greatest improvement were seen at 6 months. ${ }^{(40)}$ Moreover, the CABG patients who were enrolled in this study had the poorest HRQoL at baseline; nonetheless, large improvements were seen in this group. The questionnaire administered in this study was similar to the questionnaire utilised in the present study, hence similar results were observed. It can thus be assumed that CR encompassing lifestyle intervention, education and psychological support results in faster recovery of health and desired performance of the CABG patient. ${ }^{(27)}$ Furthermore, most CABG patients benefit from participating in a structured, comprehensive CR programme, especially when it includes components such as exercise and awareness in dealing with stress, anxiety and depression. ${ }^{(33)}$

\section{STUDY LIMITATIONS}

The main limitations of this study are the small sample size and the lack of a control group.

The sample size of this study was small for various reasons. Participants invited to partake in the research were from the Gauteng region only and some patients found it difficult to commit to every appointment because of transport and time constraints, owing to the fact that participants were required to attend 3 rehabilitation sessions a week. Only CABG patients without serious co-morbidities were considered in order to limit the number of variables.

The limited number of patients eligible for the trial led to a study design not including a control group. Each participant served as their own control with measurements being taken at the pre- and post-intervention stage.

\section{CONCLUSION}

International research has been aimed at encouraging CR combined with lifestyle education, but regardless of the many attempts to incorporate lifestyle practises into CR programmes, many health professionals are still offering one-dimensional services and individuals undergoing CABG are still under the misconception that surgery can effectively treat $C A D$, even if unaccompanied by exercise and behavioural change. This study was aimed at emphasising the value of multidisciplinary practice. By employing a lifestyle intervention programme on CABG patients, with particular emphasis on nutritional knowledge and psychosocial support, physical and mental QoL, can be improved. The findings in this research study advocate motivation to comply with a regular training programme which will enable CABG patients to benefit from overall physical and mental health.

\section{Conflict of interest: none declared.}




\section{REFERENCES}

I. Al-Hassan MA, Ahmed SM, Akhu-Zaheya LM, et al. Psychological experiences and quality of life in patient awaiting coronary artery bypass graft. International Journal of Advanced Nursing Studies 20 I 4;3(I):47-52.

2. Coyan GN, Reeder KM, Vacek JL Diet and exercise interventions following coronary artery bypass graft surgery: A review and call to action. Phys Sportsmed 2014:42(2): 1 19-29.

3. Martin F. Recognising depression after a coronary artery bypass graft. Postsurgical depression. British Journal of Nursing 2006; I (1 3):703-7.

4. Bojar R. Manual of perioperative care in adult cardiac surgery. Fourth Edition Boswell: Blackwell Science;2005.

5. Mampuya W.M. Cardiac rehabilitation past, present and future: An overview Cardiovascular Diagnosis and Therapy 2012;2(1),38-49.

6. Servey JT, Stephens M. Cardiac rehabilitation: Improving function and reducing risk. American Family Physician 2016:94(1):37-43.

7. Rantanen A, Kaunonen $\mathrm{M}$, Sintonen $\mathrm{H}$, et al. Factors associated with healthrelated quality of life in patients and significant others one month after coronary artery bypass grafting. Journal of Clinical Nursing: Blackwell Publishing;2008.

8. Burgess AW, Lerner DJ, D'Agostino RB, et al. A randomised control trial of cardiac rehabilitation. Social Science and Medicine 1987:24:359-70.

9. Oldridge NB, Guyatt $\mathrm{GH}$, Fischer M, et al. Cardiac rehabilitation after myocardial infarction: Combining data from randomised clinical trials. Journal of the American Medical Association 1988;260:945-80.

10. Alharbi M, Gallagher R, Neubeck L, et al. Exercise barriers and the relationship to self-efficacy for exercise over 12 months of a lifestyle-change program for people with heart disease and/or diabetes. European Journal of Cardiovascular Nursing 20 17;16(4):309-317.

I I. Ware JE, Kosinski M, Dewey JE. How to score version 2 of the SF- $36^{\circledR}$ Health Survey. QualityMetric Incorporated; Lincoln, Rl;2000.

12. Karachi F, Hanekom S, Faure M. Health-related quality of life of patients 12 months following surgical intensive care discharge. SA Journal of Physiotherapy 201 1;67( ( ):28-34.

13. Bolarinwa OA, Ameen HA, Sanya EO, et al. Pattern and predictive factors of health-related quality of life of patients with hypertension, diabetes and concomitant hypertension with diabetes in Ilorin, Nigeria. Nigerian Postgraduate Medical Journal 2016;23(4): I 82-190.

14. Bolarinwa OA, Juni MH, MZ NA, et al. Impact of hypertension home-based care on health related quality of life of Nigerian patients: Research concept, framework and methodology. International Journal of Public Health and Clinical Sciences 2016;3(1):|3|-|5|.

15. Paffenbarger RSAL, Wing AL, Hyde RT. Physical activity as an index of heart attack risk in college alumni. American Journal of Epidemiology 1978; 108(3): 161-75.

16. Nowak Z, Plewa M, Skowron M, Markiewicz A, Kucio C, Osiadlo G Paffenbarger physical activity questionnaire as an additional tool in clinical assessment of patients with coronary artery disease treated with angioplasty. Polish Heart Journal 201 0;68(1):32-39.

17. American College of Sports Medicine. ACSM's guidelines for exercise testing and prescription. Ninth Edition. Philadelphia: Wolters Kluwer/Lippincott Williams \& Wilkins;2014

18. Rikli RE, Jones C). Senior fitness test manual. Champaign, IL: Human Kinetics; 2001

19. Pollock ML, Welsch MA, Graves JE. Exercise prescription for cardiac rehabilitation in heart disease and rehabilitation. Champaign, IL: Human Kinetics; 1995.

20. Green SB, Salkind NJ. Using SPSS for Window and Macintosh: Analysing and understanding data. Fifth Edition. New Jersey: Pearson Prentice Hall;2008.

21. Ehrman JK, Gordon PM, Visich PS, et al. Clinical exercise physiology. Second Edition. Champaign, IL: Human Kinetics;2009.

22. Gill DL, Hammond CC, Reifsteck E), et al. Physical activity and quality of life journal of preventative medicine and public health 2013:46(I):S28-S34

23. Seguin RA, Heidkamp-Young E, Kuder J, et al. Improved physical fitness among older female participants in a nationally disseminated, communitybased exercise programme. Health Education \& Behaviour 2012;39(2): 183-90.
24. Nazari N, Javaheri SAAH, Lamir AR, et al. Effect of cardiac rehabilitation on strength and balance in patients after coronary artery bypass graft. Zahedan Journal of Research in Medical Sciences 20I3; I (15):29-33.

25. Chrysohoou C, Tsitsinakis G, Vogiatzis I, et al. High intensity, interval exercise improves quality of life of patients with chronic heart failure: A randomised controlled trial. Quarterly Journal of Medicine 20 I 3; I07(I):25-32.

26. Hsu CJ, Chen SY, Yang MC, et al. The effect of early cardiac rehabilitation on health-related quality of life among heart transplant recipients and patients with coronary artery bypass graft surgery. Transplantation Proceedings 201 I: 43:27|4-7.

27. Asghari A. The effect of alternating and continuing aerobic exercise on the systolic blood pressure and the maximum diastolic blood pressure and the resting heart rate. Research Journal of Sport Sciences 201 3; I ( I): I-7.

28. Chaudhary S, Kang MK, Sandhu JS. The effects of aerobic versus resistance training on cardiovascular fitness in obese sedentary females. Asian Journal of Sports Medicine 20 I 0; I (4): I77-84.

29. Cadore EL, Pinto RS, Pinto SS, et al. Effects of strength, endurance, and concurrent training on aerobic power and dynamic neuromuscular economy in elderly men. Journal of Strength and Conditioning Research 201 1;25(3): 756-66.

30. Aroesty JM (2010). Patient information: Recovery after coronary artery bypass graft surgery (CABG) (online). Retrieved 17 June, 201 I: http:// www. uptodate.com.

31. Adams OP. The impact of brief high-intensity exercise on blood glucose levels. Journal of Diabetes, Metabolic Syndrome and Obesity 20 1 3;6:1 I 3-22

32. Uymaz B, Sezer G, Coskun PK, et al. Clinical outcome, pain perception and activities of daily life after minimally invasive coronary artery bypass grafting. Anatolian Journal of Cardiology 20|4;14:172-7.

33. Aroesty JM (2016). Patient information: Recovery after coronary artery bypass graft surgery (CABG) (Beyond basics) (online). Retrieved 25 January, 2017:http:// www.uptodate.com.

34. Højskov IE, Moons P, Hansen, et al. SheppHeart CABG Trial - Comprehensive early rehabilitation after coronary artery bypass grafting: A protocol for a randomised clinical trial. British Medical Journal Open 2017;7:e0 I 3038 doi: I0.1 |36/bmjopen-2016-013038.

35. Coopoo Y, Berger GMB, Andrews BC. The effects of an exercise and diet programme on coronary risk factors in a sedentary Indian cohort. African Journal for Physical, Health Education, Recreation and Dance 1995; I (2):80-8

36. Shaw BS, Shaw I, Mamen A. Contrasting effects in body composition following endurance, resistance and concurrent endurance and resistance training. Journal of Sports Medicine and Physical Fitness 20 I 0;50(2):207-I 3.

37. Van Gemert WA, van der Palen J. Monninkhof EM, et al. Quality of life after diet or exercise-induced weight loss in overweight to obese postmenopausal women: The SHAPE-2 randomised controlled trial. PloS one 2015; I0(6):e0127520

38. Miyatake N, Matsumoto S, Miyachi M, et al. Relationship between changes in body weight and waist circumferences in Japanese. Environmental Health and Preventative Medicine 2007:12:220-23.

39. Ross R, Jansen I, Dawson J, et al. Exercise-induced reduction in obesity and insulin resistance in women: A randomised controlled trial. Obesity Research 2004; | 2(5):789-98.

40. Staniūtè M, Brožaitienè J. Changes in health-related quality of life among patients with coronary artery disease: A 2-year Follow-Up. Medicine (Kaunas) 2010;46(I2):843-50 